\title{
CENTIMETER COSMO-SKYMED RANGE MEASUREMENTS FOR MONITORING GROUND DISPLACEMENTS
}

\author{
F. Fratarcangeli, A. Nascetti, P. Capaldo, A. Mazzoni, M. Crespi \\ Dept. of Civil, Building and Environmental Engineering, University of Rome "La Sapienza", Via Eudossiana 18, Rome, Italy \\ < francesca.fratarcangeli,andrea.nascetti,paola.capaldo,augusto.mazzoni,mattia.crespi>@uniroma1.it
}

Commission VII, ThS 5 - 3D information extraction from SAR imagery

KEY WORDS : COSMO-SkyMed, SAR amplitude, monitoring, stereo-SAR, geophy sical effects correction

\begin{abstract}
:
The SAR (Synthetic Aperture Radar) imagery are widely used in order to monitor displacements impacting the Earth surface and infrastructures. The main remote sensing technique to extract sub-centimeter information from SAR imagery is the Differential SAR Interferometry (DInSAR), based on the phase information only. However, it is well known that DInSAR technique may suffer for lack of coherence among the considered stack of images. New Earth observation SAR satellite sensors, as COSMO-SkyMed, TerraSAR-X, and the coming PAZ, can acquire imagery with high amplitude resolutions too, up to few decimeters. Thanks to this feature, and to the on board dual frequency GPS receivers, allowing orbits determination with an accuracy at few centimetres level, the it was proven by different groups that TerraSAR-X imagery offer the capability to achieve, in a global reference frame, 3D positioning accuracies in the decimeter range and even better just exploiting the slant-range measurements coming from the amplitude information, provided proper corrections of all the involved geophysical phenomena are carefully applied. The core of this work is to test this methodology on COSMO-SkyMed data acquired over the Corvara area (Bolzano - Northern Italy), where, currently, a landslide with relevant yearly displacements, up to decimeters, is monitored, using GPS survey and DInSAR technique. The leading idea is to measure the distance between the satellite and a well identifiable natural or artificial Persistent Scatterer (PS), taking in account the signal propagation delays through the troposphere and ionosphere and filtering out the known geophysical effects that induce periodic and secular ground displacements. The preliminary results here presented and discussed indicate that COSMOSkyMed Himage imagery appear able to guarantee a displacements monitoring with an accuracy of few centimetres using only the amplitude data, provided few (at least one) stable PS's are available around the monitored area, in order to correct residual biases, likely due to orbit errors.
\end{abstract}

\section{INTRODUCTION}

The Differential SAR Interferometry (DInSAR) is the technique mainly and successfully used to extract precise information (at sub-centimetre level) from SAR data. It is well known that SAR data has a very high phase resolution, as well as the possibility to acquire images in the night and in every weather condition. This approach is widely used to monitoring the slow deformation phenomena (e.g. landslides, subsidences, volcano deformations and glacier motions) and infrastructures (e.g. buildings, dams, bridges) with the caution to study the phenomena on an image stack with a small orbital separation (baseline) in order to limit the spatial decorrelation (Berardino et al, 2002). Moreover others inconvenient of DInSAR technique are: the lack of coherence among the images stack and the complexity to unwrap the phase when the deformation phenomena are fast and much higher than the wavelength of SAR signal.

Considering these disadvantages the scientific community was brought to research a new approach to study the deformation phenomena, always with SAR data.

Currently the SAR sensors, as TerraSAR-X, COSMO-SkyMed and PAZ, have reached high resolution in amplitude also (up to
$0.20 \mathrm{~m}$ pixel resolution in the Staring SpotLight mode for TerraSAR-X and PAZ), mainly thanks to the very accurate satellite orbit (few centimetre level) due to the use of dual frequency GPS receivers on board.

Several studies were carried out using a TerraSAR-X Spotlight imagery with the purpose to evaluate the position accuracy of stable points, along the SAR line of sight, employing only the amplitude signal. In details, the position of stable and well identifiable men-made Persistent Scatterers (PS's), as corner reflectors, were estimated, in order to check at which accuracy level this approach can be used to monitor their possible displacements. The PS's position, obtained with new approach, was compared with high precision GPS surveys and the slant range measurement accuracy was found to be in the order of 10 $\mathrm{cm}$ for standard deviation and around $29 \mathrm{~cm}$ for bias (Balss et al., 2013) (Cong et al., 2012), (Eineder et al., 2011), (Gisinger et al., 2015)..

The aim of this work, which is preliminary, is the assessment and exploitation of the potential of the Italian satellite SAR sensor COSMO-SkyMed to monitoring the deformation phenomena using amplitude data in order to, in the future, to assess the capabilities to the amplitude SAR signal to monitoring the fast and large terrain displacements, which are a 
limit of the traditional and well established approach (as DInSAR). Currently, to the knowledge of the authors, there are not such a studies regarding COSMO-SkyMed.

To this aim, the same approach, already developed and applied to the image stack of TerraSAR-X acquired over Berlino area (Capaldo et al. 2014a), was applied. The article is focused on the analysis of the results; it has to be underlined that only a very short summary of the methodology is recalled, being it already well explained in the mentioned paper (Capaldo et al. 2014a).

The test field is Corvara area (Northen Italy), where an interferometric images stack (42 images) acquired by COSMOSkyMed are available. The analysed area is around $1550 \mathrm{~m}$ above sea level, at the foot of Sassongher massif, around $3 \mathrm{~km}^{2}$ of the area are affected by a fast landslide.

The paper is organized as follows: in Section 2 the methodology behind the our work is recalled; in Section 3 the dataset available and the experiments carried out are described; at the end, some conclusions are drawn in Section 4.

\section{METODOLOGY}

In the our proposed approach, only natural PS's are considered, and their approximate reference positions in a global reference frame (ECEF - Earth Centred Earth Fixed) are estimated with the stereo radargrammetric model (Capaldo et al, 2014b). The PS image coordinates are selected both on the interferometric repeat pass images stack and on the image covering the same area but acquired with different incidence angle (from the same side) in order to allow the application of stereo radargrammetric model. Since the 3D PS's position must to be very accurate and it is depend on the detection of the image coordinates, the manual collimation is not suitable to obtain high accuracy due to the not very high resolution of available SAR data and mainly due to the speckle that affect the SAR images. An automatic matching procedure has been implemented to detect the PS's image coordinates on the image; the Normalized Cross Correlation (NCC) index is used to determinate each PS position, after that an oversampling by a factor of 100x100, in range and azimuth directions respectively, was applied. Then, starting from its ECEF position and from the SAR sensor position when the PS itself is imaged, the SAR sensor to PS distance $\left(R_{\text {comp }}\right)$ is computed and it is compared with the slant-range distance measured $\left(R_{\text {meas }}\right)$ by the sensor $\left(\Delta R=R_{\text {comp }}-R_{\text {meas }}\right)$. The $S A R$ sensor position is determined on the basis of the orbital information supplied with the metadata.

The range measurements are affected by the atmospheric and geophysical effects, so that, when these effects are suitably corrected, the following equation holds:

\section{$\Delta \mathrm{R}-\mathrm{TD}+\mathrm{ID}+\mathrm{EM}=\delta+\epsilon$}

where TD is the tropospheric delay, ID the ionospheric delay, EM is the Earth surface motion (geodynamics and Earth tides), $\delta$ is the residual displacement of the PS respect to the reference estimated position and $\epsilon$ is the residual error due to the atmospheric and geophysical models errors and the remaining orbital error. Note that a possible significant non-null average of $\epsilon$ computed over different PS's imaged at the same epoch is mainly due to the common remaining orbital error and to the errors of the approximate PS computed positions; there are also minor effects due to residual error models applied for atmospheric and geophysical effects correction. The models used to correct the atmospheric delays and the free online toolkit run to evaluate the geophy sical effects are well described in (Capaldo et al. 2014b).

\section{DATASET AND EXPERIMENTS}

The available data set is formed by interferometric repeat pass COSMO-SkyMed stack of 42 Stripmap - Himage with a swath of $40 \mathrm{~km} \mathrm{x} 40 \mathrm{~km}$ and a spatial resolution of $1.56 \mathrm{~m}$ in range and $1.93 \mathrm{~m}$ in azimuth (single look), acquired from 2010 May to 2011 September in descending mode with a mean incidence angle of around 46 degree. Seventeen images were acquired by COSMO-Sky Med-1 (CSK1), twenty-three images are acquired by COSMO-SkyMed-2 (CSK2) and only two are acquired by COSMO-SkyMed-3 (CSK3) satellites and they are Level 1A products (SCS), that means they contain focused data in complex format, in slant range and zero Doppler projection. The sensor operates in X-Band, therefore with a frequency of 9.65 $\mathrm{GHz}$ and a wavelength of about 3 centimeters. The image used for the radargrammetric model is Stripmap - Himage acquired in 2010 September acquired by CSK 2 satellite with a spatial resolution of $1.00 \mathrm{~m}$ in range and $2.26 \mathrm{~m}$ in azimuth, with a mean incidence angle of around 27.5 degree, in descending mode. All image are Filtered, that indicates that the orbital data are obtained by filtering the on board navigation solution.

For the experiment, three different natural PS's are selected, they are not in the area affected by landslide, then supposed not to be affected by a displacement during the acquisition $(\delta=0)$ (Figure 1).

For the matching procedure $251 \times 251$ pixel moving window was chosen, which it is moved inside the search window of $2500 \times 2500$ pixel. It is used a large search window since the images of the interferometric stacks are not completely overlapped, being is a shift among them of around $1 \mathrm{~km}$ in azimuth. NCC's range from 0.6 to 0.9 ; low NCC's are due to speckle and not so high resolution, affecting the image matching. Then, PS positions have been computed applying the radargrammetric stereo model.

The first analy sis concerned $\Delta \mathrm{R}$ for each PS. It is important to highlight that the PS reference position is obtained as median of all PS position in the image stack; the median is considered a robust estimation of the PS reference position and the discrepancies between the median position and the PS's position on the images stack, in terms of standard deviation, is the order of around $0.8 \mathrm{~m}$ in $\mathrm{X}$ and $\mathrm{Z}$ and around 1.4 in $\mathrm{Y}$ for CSK1 (Table 1), and is the order of around $0.7 \mathrm{~m}$ in $\mathrm{X}$ and $\mathrm{Z}$ and around 1.2 in Y for CSK2 (Table 2). The 3D PS positions obtained using CSK1 images are separated from those obtained using CSK2 images, since the two sensors can be affected by different orbital errors, despite in nominal condition the satellites could be equi-phased in the same orbital plane. The results are shown in Tables 3 and 4. Only two CSK3 images are available, therefore they are not considered. 


\begin{tabular}{|c|c|c|c|c|c|c|c|c|c|}
\hline & \multicolumn{9}{|c|}{ CS K1 - Median position [m] } \\
\hline & \multicolumn{3}{|c|}{ PS1 } & \multicolumn{3}{|c|}{$\begin{array}{c}\text { PS2 } \\
\end{array}$} & \multicolumn{3}{|c|}{ PS3 } \\
\hline X-ECEF & \multicolumn{3}{|c|}{4296813.63} & \multicolumn{3}{|c|}{4287351.43} & \multicolumn{3}{|c|}{4294234.96} \\
\hline Y-ECEF & \multicolumn{3}{|c|}{902691.29} & \multicolumn{3}{|c|}{906092.28} & \multicolumn{3}{|c|}{908230.42} \\
\hline Z-ECEF & \multicolumn{3}{|c|}{4613545.84} & \multicolumn{3}{|c|}{4621353.81} & \multicolumn{3}{|c|}{4614803.83} \\
\hline & \multicolumn{9}{|c|}{ Discrepancies [m] } \\
\hline Date & $\Delta \mathbf{X}$ & $\Delta \mathbf{Y}$ & $\Delta \mathbf{Z}$ & $\Delta \mathbf{X}$ & $\Delta \mathrm{Y}$ & $\Delta \mathbf{Z}$ & $\Delta \mathbf{X}$ & $\Delta \mathbf{Y}$ & $\Delta \mathbf{Z}$ \\
\hline $17 / 06 / 10$ & 0.27 & -0.83 & 0.76 & 1.19 & -2.19 & 1.24 & 0.29 & -1.08 & 0.79 \\
\hline $03 / 07 / 10$ & 0.80 & -1.78 & 1.31 & -0.76 & 1.31 & -0.78 & 0.60 & -1.57 & 1.03 \\
\hline $19 / 07 / 10$ & 0.00 & 0.07 & 0.00 & -0.22 & 0.00 & 0.16 & 0.00 & -0.12 & 0.00 \\
\hline $04 / 08 / 10$ & -0.83 & 1.44 & -0.74 & 0.09 & -0.13 & 0.00 & -0.86 & 1.29 & -0.74 \\
\hline $20 / 08 / 10$ & -0.19 & -0.02 & 0.29 & 0.00 & 0.16 & -0.23 & -0.33 & 0.00 & 0.19 \\
\hline $05 / 09 / 10$ & 1.20 & -2.16 & 1.35 & -1.55 & 3.16 & -2.09 & 1.16 & -2.31 & 1.32 \\
\hline $21 / 09 / 10$ & 0.13 & 0.00 & -0.05 & -0.70 & 1.40 & -0.95 & 0.00 & 0.03 & -0.17 \\
\hline $23 / 10 / 10$ & 0.34 & -0.62 & 0.45 & -1.25 & 2.33 & -1.46 & 0.31 & -0.87 & 0.53 \\
\hline $08 / 11 / 10$ & 0.45 & -0.44 & 0.14 & -0.83 & 1.54 & -0.98 & 0.42 & -0.72 & 0.27 \\
\hline $24 / 11 / 10$ & 0.38 & -0.47 & 0.23 & 0.28 & -0.60 & 0.35 & 0.46 & -0.77 & 0.28 \\
\hline $01 / 04 / 11$ & -1.53 & 3.12 & -1.97 & -0.50 & 0.58 & -0.22 & -1.51 & 2.92 & -1.92 \\
\hline $17 / 04 / 11$ & -0.78 & 1.51 & -0.87 & -1.35 & 1.97 & -0.95 & -0.86 & 1.43 & -0.90 \\
\hline $03 / 05 / 11$ & -0.74 & 1.49 & -0.89 & 0.53 & -0.73 & 0.25 & -0.80 & 1.28 & -0.78 \\
\hline $19 / 05 / 11$ & 0.50 & -0.64 & 0.31 & 0.73 & -1.65 & 1.09 & 0.49 & -0.85 & 0.33 \\
\hline $04 / 06 / 11$ & -0.36 & 0.47 & -0.10 & 0.34 & -0.45 & 0.12 & -0.52 & 0.49 & -0.17 \\
\hline $06 / 07 / 11$ & -1.19 & 2.24 & -1.29 & 0.35 & -0.97 & 0.69 & -1.11 & 1.92 & -1.20 \\
\hline $22 / 07 / 11$ & -1.27 & 1.78 & -0.70 & 0.40 & -0.57 & 0.19 & -1.32 & 1.67 & -0.71 \\
\hline St.dev. & 0.76 & 1.39 & 0.86 & 0.76 & 1.41 & 0.86 & 0.75 & 1.37 & 0.82 \\
\hline
\end{tabular}

Table 1. PS's estimated positions discrepancies for CSK1

\begin{tabular}{|c|c|c|c|c|c|c|c|c|c|}
\hline & \multicolumn{9}{|c|}{ CSK2 - Median position [m] } \\
\hline & \multicolumn{3}{|c|}{ PS1 } & \multicolumn{3}{|c|}{ PS2 } & \multicolumn{3}{|c|}{ PS3 } \\
\hline X-ECEF & \multicolumn{3}{|c|}{4296807.70} & \multicolumn{3}{|c|}{4287345.59} & \multicolumn{3}{|c|}{4294229.08} \\
\hline Y-ECEF & \multicolumn{3}{|c|}{902702.15} & \multicolumn{3}{|c|}{906103.18} & \multicolumn{3}{|c|}{908241.07} \\
\hline Z-ECEF & \multicolumn{3}{|c|}{4613539.42} & \multicolumn{3}{|c|}{4621347.37} & \multicolumn{3}{|c|}{4614797.46} \\
\hline & \multicolumn{9}{|c|}{ Discrepancies [m] } \\
\hline Date & $\Delta \mathbf{X}$ & $\Delta \mathbf{Y}$ & $\Delta \mathbf{Z}$ & $\Delta \mathbf{X}$ & $\Delta \mathbf{Y}$ & $\Delta \mathbf{Z}$ & $\Delta \mathbf{X}$ & $\Delta \mathrm{Y}$ & $\Delta \mathbf{Z}$ \\
\hline $24 / 05 / 10$ & 0.85 & -1.27 & 0.58 & 0.80 & -1.39 & 0.61 & 0.81 & -1.22 & 0.63 \\
\hline $09 / 06 / 10$ & 0.97 & -1.81 & 1.09 & 0.78 & -1.57 & 0.82 & 0.93 & -1.67 & 1.02 \\
\hline $25 / 06 / 10$ & -0.79 & 1.52 & -0.98 & -0.82 & 1.51 & -1.08 & -0.73 & 1.55 & -0.95 \\
\hline $13 / 09 / 10$ & 0.78 & -1.31 & 0.70 & 0.78 & -1.42 & 0.65 & 0.82 & -1.26 & 0.67 \\
\hline $15 / 10 / 10$ & 0.48 & -0.65 & 0.24 & 0.33 & -0.59 & 0.16 & 0.49 & -0.65 & 0.30 \\
\hline $31 / 10 / 10$ & 1.05 & -1.48 & 0.62 & 0.81 & -1.24 & 0.43 & 0.97 & -1.44 & 0.72 \\
\hline $16 / 11 / 10$ & 1.27 & -1.77 & 0.74 & 1.05 & -1.63 & 0.62 & 1.27 & -1.82 & 0.85 \\
\hline $02 / 12 / 10$ & 0.65 & -1.02 & 0.50 & 0.46 & -0.85 & 0.33 & 0.81 & -1.14 & 0.54 \\
\hline $18 / 12 / 10$ & 0.23 & 0.11 & -0.37 & 0.15 & 0.11 & -0.45 & 0.41 & 0.00 & -0.34 \\
\hline $03 / 01 / 11$ & 1.21 & -1.67 & 0.69 & 1.02 & -1.53 & 0.55 & 1.21 & -1.66 & 0.74 \\
\hline 19/01/11 & 0.39 & -0.53 & 0.20 & 0.23 & -0.36 & 0.00 & 0.43 & -0.56 & 0.26 \\
\hline $04 / 02 / 11$ & 0.62 & -0.81 & 0.29 & 0.52 & -0.82 & 0.23 & 0.71 & -0.88 & 0.35 \\
\hline $20 / 02 / 11$ & -0.19 & 0.50 & -0.40 & -0.29 & 0.53 & -0.50 & -0.04 & 0.34 & -0.28 \\
\hline $08 / 03 / 11$ & -0.12 & -0.05 & 0.15 & -0.23 & 0.00 & 0.04 & 0.00 & -0.14 & 0.21 \\
\hline $24 / 03 / 11$ & -1.44 & 2.38 & -1.31 & -1.53 & 2.45 & -1.43 & -1.39 & 2.43 & -1.30 \\
\hline $09 / 04 / 11$ & -0.95 & 1.77 & -1.10 & -1.01 & 1.72 & -1.12 & -1.01 & 1.88 & -1.05 \\
\hline $25 / 04 / 11$ & 0.00 & 0.65 & -0.76 & -0.04 & 0.50 & -0.70 & -0.04 & 0.65 & -0.63 \\
\hline $11 / 05 / 11$ & -0.12 & 0.34 & -0.30 & -0.21 & 0.34 & -0.36 & -0.21 & 0.45 & -0.24 \\
\hline $27 / 05 / 11$ & -0.41 & 1.13 & -0.91 & -0.33 & 0.74 & -0.69 & -0.31 & 0.87 & -0.62 \\
\hline
\end{tabular}




\begin{tabular}{|c|c|c|c|c|c|c|c|c|c|}
\hline $12 / 06 / 11$ & -0.02 & 0.00 & 0.00 & 0.00 & -0.21 & 0.06 & -0.08 & 0.13 & 0.00 \\
\hline $28 / 06 / 11$ & -0.50 & 1.04 & -0.72 & -0.47 & 0.83 & -0.65 & -0.38 & 0.92 & -0.60 \\
\hline $30 / 07 / 11$ & -0.66 & 1.23 & -0.77 & -0.74 & 1.21 & -0.82 & -0.59 & 1.16 & -0.67 \\
\hline \hline St.dev. & $\mathbf{0 . 7 3}$ & $\mathbf{1 . 2 2}$ & $\mathbf{0 . 6 9}$ & $\mathbf{0 . 6 8}$ & $\mathbf{1 . 1 6}$ & $\mathbf{0 . 6 5}$ & $\mathbf{0 . 7 2}$ & $\mathbf{1 . 2 1}$ & $\mathbf{0 . 6 7}$ \\
\hline
\end{tabular}

Table 2. PS's estimated positions discrepancies for CSK2

\begin{tabular}{|c|c|c|c|c|c|c|c|c|c|c|c|c|}
\hline \multicolumn{13}{|c|}{ CS K1 } \\
\hline \multirow[b]{2}{*}{ Date } & \multicolumn{3}{|c|}{$\Delta \mathbf{R}[\mathrm{m}]$} & \multicolumn{2}{|c|}{$\begin{array}{c}\text { Corrections } \\
{[\mathrm{m}]}\end{array}$} & \multicolumn{3}{|c|}{$\Delta \mathrm{R}+\mathrm{EM}+\mathrm{ID}[\mathrm{m}]$} & \multirow{2}{*}{$\begin{array}{c}\text { Bias }[\mathrm{m}] \\
\epsilon_{\text {mean }}\end{array}$} & \multicolumn{3}{|c|}{$\Delta R+E M+I D-\epsilon_{\text {mean }}[\mathrm{m}]$} \\
\hline & PS 1 & PS 2 & PS 3 & ID & EM & PS 1 & PS 2 & PS3 & & PS 1 & PS 2 & PS 3 \\
\hline $17 / 06 / 10$ & 0.24 & 0.35 & 0.41 & 0.074 & -0.001 & 0.32 & 0.27 & 0.49 & 0.36 & -0.04 & -0.09 & 0.13 \\
\hline $03 / 07 / 10$ & 0.56 & 0.58 & 0.58 & 0.067 & -0.030 & 0.60 & 0.61 & 0.62 & 0.61 & -0.01 & 0.00 & 0.01 \\
\hline $19 / 07 / 10$ & -0.06 & 0.07 & 0.10 & 0.067 & -0.067 & -0.06 & 0.07 & 0.10 & 0.03 & -0.09 & 0.03 & 0.06 \\
\hline $04 / 08 / 10$ & -0.52 & -0.41 & -0.38 & 0.068 & -0.079 & -0.53 & -0.42 & -0.39 & -0.45 & -0.08 & 0.02 & 0.06 \\
\hline $20 / 08 / 10$ & -0.03 & 0.02 & 0.05 & 0.061 & -0.089 & -0.06 & -0.01 & 0.02 & -0.02 & -0.05 & 0.01 & 0.04 \\
\hline $05 / 09 / 10$ & 0.69 & 0.76 & 0.83 & 0.070 & -0.101 & 0.66 & 0.73 & 0.80 & 0.73 & -0.07 & 0.00 & 0.07 \\
\hline $21 / 09 / 10$ & -0.03 & -0.03 & 0.05 & 0.080 & -0.079 & -0.03 & -0.03 & 0.05 & 0.00 & -0.03 & -0.02 & 0.05 \\
\hline $23 / 10 / 10$ & 0.18 & 0.23 & 0.35 & 0.074 & -0.073 & 0.17 & 0.22 & 0.34 & 0.25 & -0.07 & -0.02 & 0.10 \\
\hline $08 / 11 / 10$ & 0.12 & 0.18 & 0.30 & 0.071 & -0.099 & 0.11 & 0.17 & 0.29 & 0.19 & -0.08 & -0.02 & 0.10 \\
\hline $24 / 11 / 10$ & 0.13 & 0.22 & 0.32 & 0.092 & -0.098 & 0.10 & 0.19 & 0.29 & 0.19 & -0.09 & 0.00 & 0.10 \\
\hline $01 / 04 / 11$ & -1.08 & -1.03 & -0.92 & 0.068 & -0.068 & -0.98 & -0.94 & -0.83 & -0.92 & -0.07 & -0.02 & 0.09 \\
\hline $17 / 04 / 11$ & -0.54 & -0.49 & -0.43 & 0.160 & -0.122 & -0.49 & -0.45 & -0.38 & -0.44 & -0.05 & -0.01 & 0.06 \\
\hline $03 / 05 / 11$ & -0.53 & -0.44 & -0.37 & 0.166 & -0.090 & -0.48 & -0.39 & -0.32 & -0.39 & -0.08 & 0.01 & 0.08 \\
\hline $19 / 05 / 11$ & 0.18 & 0.27 & 0.34 & 0.147 & -0.045 & 0.27 & 0.36 & 0.43 & 0.35 & -0.08 & 0.01 & 0.08 \\
\hline $04 / 06 / 11$ & -0.19 & -0.18 & -0.11 & 0.133 & -0.036 & -0.07 & -0.05 & 0.01 & -0.04 & -0.03 & -0.01 & 0.05 \\
\hline $06 / 07 / 11$ & -0.78 & -0.76 & -0.59 & 0.158 & -0.022 & -0.74 & -0.71 & -0.54 & -0.66 & -0.07 & -0.05 & 0.12 \\
\hline $22 / 07 / 11$ & -0.64 & -0.64 & -0.51 & 0.067 & -0.038 & -0.58 & -0.59 & -0.46 & -0.54 & -0.04 & -0.04 & 0.08 \\
\hline Mean & -0.14 & -0.08 & -0.00 & & & -0.11 & -0.06 & 0.03 & & -0.06 & -0.01 & 0.07 \\
\hline St.dev. & 0.47 & 0.47 & 0.46 & & & 0.45 & 0.45 & 0.44 & & 0.02 & 0.03 & 0.03 \\
\hline
\end{tabular}

Table 3. Absolute PS's accuracy assessment for CSK1

\begin{tabular}{|c|c|c|c|c|c|c|c|c|c|c|c|c|}
\hline \multicolumn{13}{|c|}{ CS K2 } \\
\hline \multirow[b]{2}{*}{ Date } & \multicolumn{3}{|c|}{$\Delta \mathrm{R}[\mathrm{m}]$} & \multicolumn{2}{|c|}{$\begin{array}{c}\text { Corrections } \\
{[\mathbf{m}]}\end{array}$} & \multicolumn{3}{|c|}{$\Delta \mathrm{R}+\mathrm{EM}+\mathrm{ID}[\mathrm{m}]$} & \multirow{2}{*}{$\begin{array}{c}\text { Bias }[\mathrm{m}] \\
\epsilon_{\text {mean }} \\
\end{array}$} & \multicolumn{3}{|c|}{$\Delta R+E M+I D-\epsilon_{\text {mean }}[\mathrm{m}]$} \\
\hline & PS 1 & PS2 & PS3 & ID & EM & PS 1 & PS2 & PS3 & & PS1 & PS2 & PS3 \\
\hline $24 / 05 / 10$ & 0.44 & 0.55 & 0.39 & 0.091 & -0.075 & 0.45 & 0.56 & 0.41 & 0.47 & -0.02 & 0.09 & -0.07 \\
\hline $09 / 06 / 10$ & 0.62 & 0.60 & 0.54 & 0.091 & -0.092 & 0.62 & 0.60 & 0.54 & 0.58 & 0.03 & 0.02 & -0.05 \\
\hline $25 / 06 / 10$ & -0.50 & -0.43 & -0.54 & 0.068 & -0.105 & -0.54 & -0.47 & -0.58 & -0.53 & -0.01 & 0.06 & -0.05 \\
\hline $13 / 09 / 10$ & 0.45 & 0.55 & 0.40 & 0.068 & -0.098 & 0.42 & 0.52 & 0.37 & 0.44 & -0.02 & 0.09 & -0.07 \\
\hline $15 / 10 / 10$ & 0.23 & 0.28 & 0.19 & 0.078 & -0.050 & 0.26 & 0.30 & 0.22 & 0.26 & 0.00 & 0.04 & -0.04 \\
\hline $31 / 10 / 10$ & 0.51 & 0.49 & 0.46 & 0.066 & -0.020 & 0.55 & 0.54 & 0.51 & 0.53 & 0.02 & 0.01 & -0.03 \\
\hline $16 / 11 / 10$ & 0.61 & 0.63 & 0.59 & 0.063 & 0.020 & 0.69 & 0.71 & 0.67 & 0.69 & 0.00 & 0.02 & -0.02 \\
\hline $02 / 12 / 10$ & 0.35 & 0.36 & 0.36 & 0.066 & 0.049 & 0.47 & 0.48 & 0.48 & 0.47 & -0.01 & 0.00 & 0.00 \\
\hline $18 / 12 / 10$ & -0.02 & 0.05 & -0.02 & 0.064 & 0.038 & 0.08 & 0.15 & 0.08 & 0.10 & -0.02 & 0.04 & -0.02 \\
\hline $03 / 01 / 11$ & 0.58 & 0.59 & 0.54 & 0.073 & -0.038 & 0.61 & 0.63 & 0.57 & 0.60 & 0.01 & 0.02 & -0.03 \\
\hline $19 / 01 / 11$ & 0.19 & 0.20 & 0.17 & 0.059 & -0.074 & 0.17 & 0.18 & 0.15 & 0.17 & 0.01 & 0.01 & -0.02 \\
\hline $04 / 02 / 11$ & 0.29 & 0.35 & 0.27 & 0.064 & -0.089 & 0.26 & 0.33 & 0.25 & 0.28 & -0.02 & 0.05 & -0.03 \\
\hline $20 / 02 / 11$ & -0.16 & -0.10 & -0.14 & 0.118 & -0.088 & -0.13 & -0.07 & -0.11 & -0.10 & -0.03 & 0.03 & 0.00 \\
\hline $08 / 03 / 11$ & 0.02 & 0.07 & 0.02 & 0.180 & -0.040 & 0.16 & 0.21 & 0.16 & 0.18 & -0.02 & 0.03 & -0.02 \\
\hline $24 / 03 / 11$ & -0.79 & -0.75 & -0.84 & 0.110 & 0.062 & -0.62 & -0.57 & -0.67 & -0.62 & 0.00 & 0.05 & -0.05 \\
\hline $09 / 04 / 11$ & -0.59 & -0.50 & -0.66 & 0.172 & 0.058 & -0.36 & -0.27 & -0.43 & -0.35 & 0.00 & 0.08 & -0.08 \\
\hline $25 / 04 / 11$ & -0.20 & -0.09 & -0.24 & 0.146 & 0.062 & 0.01 & 0.12 & -0.03 & 0.03 & -0.03 & 0.09 & -0.06 \\
\hline $11 / 05 / 11$ & -0.10 & -0.04 & -0.18 & 0.147 & 0.053 & 0.10 & 0.16 & 0.02 & 0.09 & 0.00 & 0.07 & -0.07 \\
\hline
\end{tabular}




\begin{tabular}{|c|c|c|c|c|c|c|c|c|c|c|c|c|}
\hline $27 / 05 / 11$ & -0.37 & -0.17 & -0.32 & 0.092 & -0.021 & -0.29 & -0.10 & -0.24 & -0.21 & -0.08 & 0.11 & -0.03 \\
\hline $12 / 06 / 11$ & 0.01 & 0.15 & -0.07 & 0.113 & -0.077 & 0.04 & 0.18 & -0.03 & 0.06 & -0.02 & 0.12 & -0.10 \\
\hline $28 / 06 / 11$ & -0.34 & -0.20 & -0.33 & 0.113 & -0.083 & -0.31 & -0.17 & -0.30 & -0.26 & -0.05 & 0.09 & -0.04 \\
\hline $30 / 07 / 11$ & -0.40 & -0.33 & -0.42 & 0.092 & -0.108 & -0.42 & -0.35 & -0.43 & -0.40 & -0.02 & 0.05 & -0.03 \\
\hline $15 / 08 / 11$ & -0.37 & -0.31 & -0.44 & 0.065 & -0.088 & -0.40 & -0.33 & -0.47 & -0.40 & 0.00 & 0.07 & -0.07 \\
\hline \hline Mean & $\mathbf{0 . 0 2}$ & $\mathbf{0 . 0 8}$ & $\mathbf{- 0 . 0 1}$ & & & $\mathbf{0 . 0 8}$ & $\mathbf{0 . 1 5}$ & $\mathbf{0 . 0 5}$ & & $\mathbf{- 0 . 0 1}$ & $\mathbf{0 . 0 5}$ & $\mathbf{- 0 . 0 4}$ \\
\hline St.dev. & $\mathbf{0 . 4 1}$ & $\mathbf{0 . 3 9}$ & $\mathbf{0 . 4 1}$ & & & $\mathbf{0 . 3 9}$ & $\mathbf{0 . 3 7}$ & $\mathbf{0 . 3 9}$ & & $\mathbf{0 . 0 2}$ & $\mathbf{0 . 0 3}$ & $\mathbf{0 . 0 2}$ \\
\hline
\end{tabular}

Table 4. Absolute PS's accuracy assessment for CSK2

The dispersion, in terms of standard deviation, between the slant range measurements, is close to 0.47 and $0.40 \mathrm{~m}$ for the two satellites CSK1, CSK2 respectively.

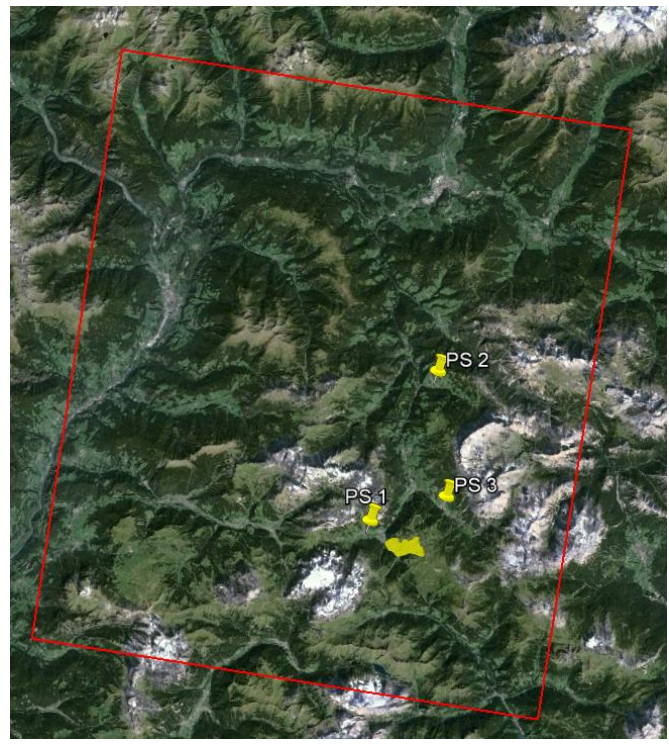

Figure 1. PS's (PS 1,2,3) distribution and landslide (yellow area) position on the COSMO-SkyMed image

The radar measurements have been corrected taking into account of atmospheric delays and geophysical effects and one more time compared with 3D distance between the reference PS position and satellite position. In detail since the hourly ZTD estimated at the Bolzano GNSS station (BZRG), that is the permanent station closest to image area is around $2.2 \mathrm{~m}$, it can be inferred that the COSMO-SkyMed signal are already corrected for the tropospheric delay, but the model is not declared. The ionospheric delay reaches at maximum 18 centimeters and the geophysical effect 6 centimeters both along the SAR line of sight. The new results after the slant range corrections are summarized again in Tables 3 and 4.

The applied slant range corrections (ID and EM) are rather small (within an absolute value of $18 \mathrm{~cm}$ ), so that the results display improvements of the standard deviations limited to a couple of centimeters (from 0.47 to $0.45 \mathrm{~m}$ for CSK 1 and from 0.40 to 0.38 for CSK2). It is possible to note that the PS position discrepancies in the same acquisition, after range corrections, are affected by a significant non-null bias (up to several decimetres, in absolute values), probably mainly due to orbital errors, as previously mentioned. These biases can be removed (under the hypothesis of stable PS's), what remarkably improves the accuracy of range measurements up to few centimeters (standard deviation of $2-3 \mathrm{~cm}$ and maximum bias of $7 \mathrm{~cm}$ ).

\section{CONCLUSIONS}

The aim of this preliminary work was the preliminary exploitation of the potential of the Italian satellite SAR sensor COSMO-SkyMed to monitor displacement using amplitude data. A methodology already developed and applied to TerraSAR-X imagery (Capaldo et Al., 2014), was tested on a set of 42 COSMO-SkyMed Himage imagery acquired over the area of Corvara (Bolzano, Northern Italy) from 2010 and 2011 with an mean incidence angle of 46 degrees and one single image acquired in 2010 with a mean incidence angle of 27 degree. Three different, stable and natural PS's have been selected. The slant range accuracy assessments of three stable PS's highlight a standard deviation of $2-3 \mathrm{~cm}$ and a bias at maximum level of 7 $\mathrm{cm}$.

Therefore the COSMO-SkyMed Himage imagery appear able to guarantee a displacements monitoring with an accuracy of few centimetres using only the amplitude data, provided few (at least one) stable PS's are available around the monitored area, in order to correct the biases mainly due to orbit errors.

In the future the assessment of these capabilities will be developed considering corner reflectors located on the Corvara landslide, impacted by fast and high terrain displacements (up to decimetres/meters per year).

\section{ACKNOWLEDGEMENTS}

The authors thank very much Dr. Luca Pietranera from e-Geos (Rome, Italy) for making available the stereo radargrammetric COSMO-SkyMed image, and Dr. Marco Mulas from the University of Modena and Reggio Emilia, for making available the interferometric COSMO-SkyMed images stack.

\section{REFERENCES}

Balss, U., Gisinger, C., Cong, X.Y., Eineder, M., Brcic, R., 2013. Precise 2-D and 3-D ground target localization with TerraSAR-X. International Archives of the Photogrammetry, Remote Sensing and Spatial Information Sciences. Vol. XL1/W1, pp. 23-28.

Berardino, P., Fornaro, G., Lanari, R., Sansosti, E., 2002. A new algorithm for surface deformation monitoring on small baseline 
differential SAR interferograms. IEEE Transactions on Geoscience and Remote Sensing 40(11), pp. 2375-2383.

Capaldo, P., Fratarcangeli, F., Nascetti, A., Mazzoni, A., Porfiri, M., Crespi, C., 2014a. Centimeter range measurement using amplitude data of TerraSAR-X imagery. The International Archives of the Photogrammetry, Remote Sensing and Spatial Information Science, XL-7, 55-61, ISPRS Technical Commission VII Symposium, 29 September - 2 October 2014, Istanbul, Turkey.

Capaldo, P., Nascetti, A., Porfiri, M., Pieralice, F., Fratarcangeli, F., Crespi, C., Toutin, T., 2014b. Evaluation and comparison of different radargrammetric approaches for the Digital Surface Models generation from COSMO-SkyMed, TerraSAR-X, RADARSAT-2 imagery: analysis of Beauport (Canada) test site. ISPRS Journal of Photogrammetry and Remote Sensing, Vol.100, pp.60-70.

Cong, X., Balss, U., Eineder, M., Fritz, T., 2012. Imaging Geodesy Centimeter-Level Ranging Accuracy With TerraSAR$\mathrm{X}$ : An Update. IEEE Transactions on Geoscience and Remote Sensing, vol. 9, n.5, pp. 948-952.

Eineder, M., Minet, C., Steigenberger, P., Cong, X., Fritz, T., 2011. Imaging geodesy - Toward centimeter-level ranging accuracy with TerraSAR-X. IEEE Transactions on Geoscience and Remote Sensing. Vol.49, n. 2, pp. 661-671.

Gisinger, C., Gernhardt, S., Auer, S., Balss, U., Hackel, S., Pail, R., Eineder, M., 2015. Absolute 4-D positioning of persistent scatterers with TerraSAR-X by applying geodetic stereo SAR. IEEE International Geoscience and Remote Sensing Symposium (IGARSS). pp. $2991-2994$. 